Onkologie 1987;10(suppl 1):3

\title{
Impressum, Vol. 10, Supplement 1, 1987
}

\section{s. karger}

Verlag für Medizin und Naturwissenschaften GmbH, Postfach 1724, D-8034 Germering

Presserechtlich verantwortlich: Walter Kunz, Gesellschafter

Wissenschaftlicher Beirat

H. W. Bauer, Berlin; W. Bossnew, Sofia/Bulgarien; H. Denck, Wien; V. Diehl, Köln; P. Drings, Heidelberg; E. Dühmke, Göttin-gen; S. Eckhardt, Budapest; H. Ehrhart, München; H. H. Fiebig, Freiburg; W. M. Gallmeier, Nürnberg; E. H. Graul, Marburg/ Lahn; R. Hartenstein, München; H. Heimpel, Ulm; K. P. Hellrie-gel, Berlin; Ch. Herfarth, Heidelberg; H. W. von Heyden, Ein-beck; D. Hoelzer, Frankfurt/M.; J. H. Holzner, Wien; R. Hünig, Basel; W. Hunstein, Heidelberg; H. J. Illiger, Oldenburg; U. R. Kleeberg, Hamburg; H. O. Klein, Köln; B. Kornhuber, Frankfurt/M.; H. Löffler, Kiel; U. Mohr, Hannover; K. Munk, Heidelberg; G. A. Nagel, Göttingen; J. P. Obrecht, Basel; A. Pfleiderer, Freiburg; K. Possinger, München; W. Queißer, Mannheim; H. Riehm, Hannover; E. Scherer, Essen; M. Schroder, Göttingen; S. Seeber, Leverkusen; H. J. Senn, St. Gallen; A. Stacher, Wien; V. Sturm, Heidelberg; St. Tanneberger, Berlin/DDR; W. Vahlensieck, Bonn; H. D. Waller, Tubingen; M. Wannen-macher, Freiburg; W. Wilmanns, München; K.

Wilms, Würzburg; K. zum Winkel, Heidelberg; H. Wrba, Wien.

Schriftleitung: S. Eckhardt, Budapest J. H. Holzner, Wien G. A. Nagel, Göttingen

Anzeigen

S. Karger Verlag für Medizin und Naturwissenschaften GmbH,

Postfach 1724, D-8034 Germering, Telefon (089) 844021.

Für den Inhalt außerhalb des redaktionellen Teiles (insbesondere Anzeigen,

Industrieinformationen, Pressezitate und Kongreßinfor-mationen) übernehmen Herausgeber und Verlag keine Gewähr.

Eine Markenbezeichnung kann warenzeichenrechtlich geschützt sein, auch wenn bei ihrer Verwendung in dieser Zeitschrift das Zeichen ${ }^{\circledR}$ oder ein anderer Hinweis auf etwa bestehende Schutz-rechte fehlen sollte.

Alle Rechte, insbesondere das Recht der Vervielfältigung und Mikrokopie sowie der Übersetzung, vorbehalten Nachdruck, auch auszugsweise, nur mit Genehmigung des Verlages. (c) Copyright 1987 by S. Karger Verlag für Medizin und Naturwissenschaften GmbH, Postfach 1724, D-8034 Germering ISBN 3-8055-4588-6

Satz und Druck: Walter Biering GmbH, Grafischer Betrieb, 8000 München 45

Redaktionskomítee dieses Sonderheftes

H. Sauer, W. Eiermann, K. Possinger, G. Ries, H. Schünemann, N. Willich

Die weiteren Mitglieder der Projektgruppe Mammakarzinom und deren Adressen sind ab S. 43 aufgeführt.

Ladenpreis dieses Sonderheftes DM 24,- incl. MwSt. Abonnenten erhalten es unberechnet. Bezugspreis der Zeitschrift:

Für Jahrgang 10, 1987, DM 148,-/öS 1036,-/SFr. 116, 1 Einzelheft kostet DM 28,-/öS 196,-/SFr. 22.-, einschließlich MwSt., zuzüglich Postgebühren. 1987;010:3 
